\title{
LEARNINGS FROM EXERCISE IS MEDICINE CANADA WORKSHOPS AS STRATEGIES TO ASSIST MEDICAL STUDENTS PROMOTE PHYSICAL ACTIVITY DURING AND BEYOND THE COVID-19 PANDEMIC
}

\author{
M.W. O'Brien, MSc a*, M.Y.S. Wong, MSc ${ }^{\mathrm{b} *}$,W. Sui, PhD', \\ M.L. Voss, MSc ${ }^{\mathrm{d}}$, N.W. Bray, MSc ${ }^{\mathrm{b}}$, C.N. Turnbull, BKin ${ }^{\mathrm{e}}$, \\ T.S. Nagpal, PhD $\mathrm{f}, \mathrm{g}$, and J.R. Fowles, $P h D^{\mathrm{h}}$
}

\section{ABSTRACT}

Despite the health benefits of movement, physical activity (PA) promotion is insufficiently covered in medical training programmes. Given the decreases in the populations PA level from the coronavirus pandemic, PA educational training is needed in medical school now more than ever. Exercise is Medicine Canada (EIMC) has designed and delivered workshops that improve physicians' exercise prescription practices. The learning objectives of the workshop include (i) discuss the health benefits of PA with patients, (ii) monitor PA as a vital sign, (iii) provide basic exercise counselling and prescription, (iv) utilize a motivational counselling approach and (v) monitor aerobic and

\footnotetext{
a Division of Kinesiology, Dalhousie University, Nova Scotia, Canada

b School of Kinesiology, Faculty of Health Sciences, Western University, Ontario, Canada

c School of Exercise Science, Physical and Health Education, University of Victoria, British Columbia, Canada

d Faculty of Health Sciences, University of Lethbridge, Alberta, Canada

e Faculty of Kinesiology and Recreation Management, University of Manitoba, Manitoba, Canada

f School of Human Kinetics, University of Ottawa, Ontario, Canada

g Society of Obstetricians and Gynaecologists of Canada, Ontario, Canada

h School of Kinesiology, Acadia University, Nova Scotia, Canada

* Authors contributed equally.
} 
resistance exercise adherence. We propose that the workshop learning objectives may be used as competencies for future medical education. A greater integration of PA education into medical school curriculum and continuing education opportunities may help combat the rising population rates of physical inactivity during and beyond the coronavirus pandemic to improve population health.

Keywords: physical activity, exercise is medicine, medical school, curriculum, COVID-19

Corresponding Author: Myles William O'Brien, School of Health and Human Performance, Division of Kinesiology, Dalhousie University, 6230 South Street, Halifax, Nova Scotia, Canada, B3H 4R2 Email: myles.obrien@dal.ca

\section{BACKGROUND}

The coronavirus (COVID-19) pandemic has dramatically changed daily life, and in turn has detrimentally impacted physical and mental well-being (United Nations Policy Brief, 2020; Wang et al., 2020). Decreasing levels of physical activity (PA) have been observed worldwide (Guthold et al., 2018), which may be exacerbated by COVID-19 restrictions (Tison et al., 2020) through the closure of businesses, schools, exercise facilities and green spaces. Such restrictions and limitations on traditional opportunities to engage in physical activity are problematic, as the health benefits of engaging in regular PA are well-documented (Pedersen and Saltin, 2015) and inactivity is associated with a higher risk for severe COVID-19 outcomes (Sallis et al., 2021).

Physicians report low confidence in their ability to prescribe exercise to their patients, as well as experience a number of impactful barriers that discourage the inclusion of PA in their practice, including lack of personal knowledge, billing structure, time constraints, perceived low patient interest and awareness of available resources (Swinburn et al., 1997; O'Brien et al., 2017). Together, this results in few physicians $(\sim 16 \%)$ providing patients with PA counselling and written exercise prescriptions (Petrella et al., 2003). Increased physician burnout (West, Dyrbye, and Shanafelt, 2018, Amanullah and Ramesh, 2020) and health care system stress during the pandemic have likely compounded these barriers to push the promotion of healthy lifestyle 
behaviours to the 'back burner'. Now, more than ever, there is a need to increase the promotion of physical activity by healthcare professionals to improve population health and well-being (Natalucci et al., 2020) both during and after the COVID-19 pandemic. Importantly, low PA is associated with higher mortality (Ross et al., 2016) and more severe complications from COVID-19 (Sallis et al., 2021).

Including PA content in medical school curricula may be a suitable intervention point to increase physician comfort with PA promotion. Specifically, $85 \%$ of Canadian medical students perceived their PA counselling and exercise prescription education as insufficient (Solmundson, Koehle, and McKenzie, 2016). Similar observations were observed in medical schools in the United Kingdom, with undergraduate medical students reporting low confidence in their ability to include physical activity with patients despite recognising that advising patients about physical activity is an important part of a physician's job (Pugh et al., 2020). In the United Kingdom, only 4.2 hours of the undergraduate curriculum is spent on physical activity science and promotion (Weiler et al., 2012) and most students in their final year of medical school $(80 \%)$ state they have not received any training in lifestyle medicine in the last two years, despite $76 \%$ desiring more lifestyle medicine education (Radenkovic et al., 2019). Together, these statistics highlight an area for improvement, which could positively affect patient outcomes once these students become practising medical professionals. For example, exercise prescriptions by physicians who receive some exercise prescription training have been shown to increase patients' physical activity and physical fitness levels, and improve health outcomes, such as lowering systolic blood pressure and body mass index (Petrella et al., 2003; Grandes et al., 2009). Even simple, brief strategies that promote the discussion of PA in patient-provider appointments such as a 'physical activity vital sign' (Sallis 2011) can change both patient and provider behaviour (Grant et al., 2014).

\section{WHAT WE DID}

Exercise is Medicine ${ }^{\circledR}$ is an international initiative aimed at increasing the number of health care providers assessing, counselling and prescribing PA as part of routine healthcare visits (Exercise is Medicine Canada, 
2021). To fulfil this mission, Exercise is Medicine Canada (EIMC) designed and launched full-day educational workshops to physicians across Canada. The purpose of the present article is to highlight the successes of the EIMC workshops on physicians' inclusion of activity into their practice and position the learning objectives of the workshop as basic competencies for medical curriculum design and delivery.

\section{HOW WE DID IT}

The interactive full-day workshop was delivered across seven provinces in Canada (Fowles et al., 2018). The effectiveness of the EIMC workshop among Canadian physicians was determined via a self-report questionnaire assessing their physical activity perceptions and practices prior to (O'Brien et al., 2017) and three-months post-workshop (Fowles et al., 2018). The learning objectives of the EIMC workshop expand upon previous, shorter (i.e., three-hour) educational workshops for physicians that included: how to assess a patient's physical activity levels, using motivational interviewing techniques to increase patients' physical activity, and providing written exercise prescriptions when appropriate (Windt et al., 2015). Specifically, the EIMC workshop covered five learning objectives, as presented in Table 1 (O’Brien et al., 2017).

Table 1. Learning objectives of the Exercise is Medicine Canada interactive workshop

\begin{tabular}{|l|l|}
\hline Number & Learning Objective \\
\hline 1 & $\begin{array}{l}\text { Discuss the health benefits and safety of regular exercise } \\
\text { with patients }\end{array}$ \\
\hline 2 & $\begin{array}{l}\text { Be able to determine a patients' physical activity or exercise } \\
\text { vital sign (i.e., time spent engaging in moderate-vigorous } \\
\text { physical activity per week) }\end{array}$ \\
\hline 3 & $\begin{array}{l}\text { Provide basic exercise counselling and prescription for } \\
\text { patients as part of the periodic health evaluation }\end{array}$ \\
\hline 4 & $\begin{array}{l}\text { Utilize a motivational counselling framework for health } \\
\text { behaviour change }\end{array}$ \\
\hline 5 & $\begin{array}{l}\text { Understand how to monitor aerobic exercise intensity and } \\
\text { perform basic resistance exercises }\end{array}$ \\
\hline
\end{tabular}




\section{WHAT WE HAVE LEARNED}

Overall, physicians' confidence to provide physical activity counselling increased from $\sim 50 \%$ to $\sim 75 \%$, with the rates of physicians prescribing exercise increasing from $20 \%$ to $74 \%$ three-months following the workshop (Fowles et al., 2018). To discern whether physicians' initial confidence level influenced the effectiveness of the training, a subsequent study evaluated the effectiveness of the workshop across physicians with low, medium and high pre-workshop confidence levels for incorporating PA into patient visits. After training, all groups' self-confidence to include physical activity with patients significantly improved (relative increase: low: $+40 \%$, medium: $+20 \%$, high: $+10 \%$ ) and they increased the frequency of providing written exercise prescriptions to patients (low: $10 \%$ to $62 \%$, medium: $16 \%$ to $89 \%$, high: $57 \%$ to $87 \%$; O'Brien et al., 2020). Of note, physicians with low pre-workshop confidence identified 'a lack of exercise education in medical school' as one of the most impactful barriers initially preventing the inclusion of physical activity into their practice (O'Brien et al., 2020).

Notably, we learned that an interactive workshop can change physicians' clinical practice, particularly among medical providers with low initial confidence that cite insufficient training on the topic in medical school; in other words, those who need the educational training the most. Accordingly, we propose that educational opportunities, such as medical design and curricula, should consider basing their framework for including more physical activity content on the learning objectives of our EIMC workshop that has demonstrated success. We suggest every medical school curriculum should have at least 3.5 hours of coverage on the topic, preferably seven hours, that includes both lecture and in-person interactive components that include case studies of PA counselling scenarios and exercise demonstration, delivered by exercise specialists or physicians with Kinesiology backgrounds. Providing continuing medical education on PA for physicians in practice should be a standard offering.

\section{TAKE HOME POINTS}

Prior to the COVID-19 pandemic, our increasingly sedentary population and lack of PA training for medical professionals were already concerns. The immediate and predicted long-term impacts of COVID-19 on the 
healthcare system and individuals' health and well-being highlight the need for physical activity promotion to become a standard part of medical care. Adjusting existing medical curricula and providing more continuing education on this topic for practising physicians may increase physician knowledge around the promotion of physical activity. We provide five major learning objectives used in our EIMC workshops that have demonstrated efficacy among physicians. We propose that these objectives may serve as an ideal starting point for medical programmes to use as competencies in an effort to promote greater inclusion of physical activity into medical training and relay the importance of physical activity for patients' overall well-being. With frequent and continued discussions about physical activity, healthcare professionals can play an important role in reversing the current trends of increasing chronic disease and declining overall well-being that have resulted from physical inactivity caused by the COVID-19 pandemic (Tison et al., 2020).

\section{ACKNOWLEDGEMENTS AND DISCLOSURES}

Funding: MWO was supported by a Heart \& Stroke Bright Red Scholarship, Nova Scotia Graduate Scholarship, a Research Nova Scotia Scotia Scholars Award, a Killam PreDoctoral Scholarship, and a Fredrick Banting and Charles Best CIHR Doctoral Award. TSN is funded by Mitacs supported by The Society of Obstetricians and Gynaecologists of Canada. Authors are members of the Exercise is Medicine Canada student committee (MWO, MYSW, WS, MLV, NWB, CNT, TSN).

Author Contributions: MWO, MYSW and WS drafted the manuscript. All authors edited and revised the document. All authors approved the final version of the manuscript.

Competing Interests: JRF is the National Chair of Exercise is Medicine Canada.

\section{REFERENCES}

Amanullah, S. and Ramesh Shankar R., 2020. The impact of COVID-19 on physician burnout globally: a review. Healthcare, 8(4), pp. 421. https://doi.org/10.3390/healthcare8040421 
Exercise is Medicine Canada. 2021. Available at: https://exerciseismedicine .org/canada [accessed 22 January 2021].

Fowles, J.R., O'Brien, M.W., Solmundson, K., Oh, P.I. and Shields, C.A., 2018. Exercise is Medicine Canada physical activity counselling and exercise prescription training improves counselling, prescription, and referral practices among physicians across Canada. Applied Physiology, Nutrition, and Metabolism, 43(5), pp. 535-539. https://doi.org/10.1139/apnm-2017-0763

Grandes, G., Sanchez, A., Sanchez-Pinilla, R.O., Torcal, J., Montoya, I., Lizarraga, K. and Serra, J. 2009. Effectiveness of physical activity advice and prescription by physicians in routine primary care: a cluster randomized trial. Archives of Internal Medicine, 169(7), pp. 694-701. https://doi.org/10.1001/archinternmed.2009.23

Grant, R.W., Schmittdiel, J.A., Neugebauer, R.S., Uratsu, C.S. and Sternfeld, B., 2014. Exercise as a vital sign: a quasi-experimental analysis of a health system intervention to collect patient-reported exercise levels. Journal of General Internal Medicine, 29(2), pp. 341-348. https://doi.org/10.1007/s11606-013-2693-9

Guthold, R., Stevens, G.A., Riley, L.M. and Bull, F.C., 2018. Worldwide trends in insufficient physical activity from 2001 to 2016: a pooled analysis of 358 population-based surveys with 1.9 million participants. The Lancet Global Health, 6(10), pp. e1077-e1086. https://doi.org/10.1016/S2214-109X(18)30357-7

Natalucci, V., Pellino, V.C., Barbieri, E. and Vandoni, M., 2020. Is it important to perform physical activity during coronavirus pandemic (COVID-19)? Driving action for a correct exercise plan. Frontiers in Public Health, 8. https://doi.org/10.3389/ fpubh.2020.602020

O'Brien, M.W., Shields, C.A., Oh, P.I. and Fowles, J.R., 2017. Health care provider confidence and exercise prescription practices of Exercise is Medicine Canada workshop attendees. Applied Physiology, Nutrition, and Metabolism, 42(4), pp. 384-390. https:// doi.org/10.1139/apnm-2016-0413

O'Brien, M.W., Shields, C.A., Solmundson, K. and Fowles, J.R., 2020. Exercise is Medicine Canada workshop training improves physical activity practices of physicians across Canada, independent of initial confidence level. Canadian Medical Education Journal, 11(5), pp. e5. https://doi.org/10.36834/cmej.68376 
Pedersen, B.K. and Saltin, B. 2015. Exercise as medicine-evidence for prescribing exercise as therapy in 26 different chronic diseases. Scandinavian Journal of Medicine \& Science in Sports, 25, pp. 1-72. https://doi.org/10.1111/sms.12581

Petrella, R.J., Koval, J.J., Cunningham, D.A. and Paterson, D.H., 2003.

Can primary care doctors prescribe exercise to improve fitness? The step test exercise prescription (STEP) project. American Journal of Preventive Medicine, 24(4), pp. 316-322. https://doi. org/10.1016/s0749-3797(03)00022-9

Pugh, G., O'Halloran, P., Blakey, L., Leaver, H. and Angioi, M., 2020. Integrating physical activity promotion into UK medical school curricula: testing the feasibility of an educational tool developed by the Faculty of Sports and Exercise Medicine. BMJ Open Sport \& Exercise Medicine, 6(1), pp. e000679. https://doi. org/0.1136/bmjsem-2019-000679

Radenkovic, D., Aswani, R., Ahmad, I., Kreindler, J. and Robinson, R., 2019. Lifestyle medicine and physical activity knowledge of final year UK medical students. BMJ Open Sport \& Exercise Medicine, 5(1), pp. e000518. http://dx.doi.org/10.1136/bmjsem-2019 $-000518$

Ross, R., Blair, S.N., Arena, R., Church, T.S., Després, J.P., Franklin, B.A., Haskell, W.L., Kaminsky, L.A., Levine, B.D., Lavie, C.J., Myers, J., Niebauer, J., Sallis, R., Sawada, S.S., Sui, X. and Wisløff, U., 2016. Importance of assessing cardiorespiratory fitness in clinical practice: A case for fitness as a clinical vital sign: A scientific statement from the American Heart Association. Circulation, 134(24), pp. e653-e699. https://doi.org/10.1161/ CIR.0000000000000461

Sallis, R., 2011. Developing healthcare systems to support exercise: exercise as the fifth vital sign. British Journal of Sports Medicine, 45(6), pp. 473-474. https://doi.org/10.1136/bjsm.2010.083469

Sallis, R., Young, D.R., Tartof, S.Y., Sallis, J.F., Sall, J., Li, Q., Smith, G.N. and Cohen, D.A., 2021. Physical inactivity is associated with a higher risk for severe COVID-19 outcomes: a study in 48440 adult patients. British Journal of Sports Medicine, In Press. https:// doi.org/10.1136/bjsports-2021-104080 
Solmundson, K., Koehle, M. and McKenzie, D., 2016. Are we adequately preparing the next generation of physicians to prescribe exercise as prevention and treatment? Residents express the desire for more training in exercise prescription. Canadian Medical Education Journal, 7(2), pp. e79.

Swinburn, B.A., Walter, L.G., Arroll, B., Tilyard, M.W. and Russell, D.G., 1997. Green prescriptions: attitudes and perceptions of general practitioners towards prescribing exercise. British Journal of General Practice, 47(422), pp. 567-569.

Tison, G.H., Avram, R., Kuhar, P., Abreau, S., Marcus, G.M., Pletcher, M.J. and Olgin, J.E., 2020. Worldwide effect of COVID-19 on physical activity: a descriptive study. Annals of Internal Medicine, 173(9), pp. 767-770. https://doi.org/10.7326/M20-2665

United Nations Policy Brief., 2020. COVID-19 and the need for action on mental health. Available at: https://unsdg.un.org/resources /policy-brief-covid-19-and-need-action-mental-health [accessed 22 January 2021].

Wang, C., Pan, R., Wan, X., Tan, Y., Xu, L., McIntyre, R.S., Choo, F.N., Tran, B., Ho, R., Sharma, V.K. and Ho, C., 2020. A longitudinal study on the mental health of general population during the COVID-19 epidemic in China. Brain, Behavior, and Immunity, 87, pp. 40-48. https://doi.org/10.1016/j.bbi.2020.04.028

Weiler, R., Feldschreiber, P. and Stamatakis, E., 2012. Medicolegal neglect? The case for physical activity promotion and exercise medicine. British Journal of Sports Medicine, 46(4), pp. 228-232. http://dx.doi.org/10.1136/bjsm.2011.084186

West, C.P., Dyrbye, L.N. and Shanafelt, T.D. 2018. Physician burnout: contributors, consequences and solutions. Journal of Internal Medicine, 283(6), pp. 516-529. https://doi.org/10.1111/joim.12752

Windt, J., Windt, A., Davis, J., Petrella, R. and Khan, K., 2015. Can a 3-hour educational workshop and the provision of practical tools encourage family physicians to prescribe physical activity as medicine? A pre-post study. BMJ Open, 5(7). http://dx.doi. org/10.1136/bmjopen-2015-007920 